\title{
On fusion algebra of chiral $S U(N)_{K}$ models
}

\author{
A Lima-Santos \\ Departamento de Fisica, Universidade Federal de São Carlos, Caixa Postal 676, 13569- \\ 905 São Carlos, Brazil
}

\begin{abstract}
We discuss some algebraic setting of chiral $S U(N)_{K}$ models in terms of the satistical dimensions of their fields. In particular, the conformal dimensions and the central charge of the chiral $S U(N)_{K}$ models are calculated from their braid matrices. Furthermore, at level $K=2$, we present the characteristic polynomials of their fusion matrices in a factored form.
\end{abstract}

\section{Introduction}

Fusion algebras are found to play an important role in the study of rational conformal field theories (RCFT). Beside the fact that the fusion rules can be expressed in terms of the unitary matrix $S$ [ [] that encodes the modular transformations of the characters of the RCFT

$$
N_{i j}^{k}=\sum_{l} \frac{S_{i l}}{S_{0 l}} S_{j l} S_{k l}^{*}
$$

where " 0 " refers to the identity operator, and the labels $i, \ldots, l$ run over $n$ values corresponding to the primary fields of the chiral algebra of the RCFT, there is a more fundamental reason to look for representations of the fusion algebra, based on the concept of operator products [2]. When one tries to compute the operator product coefficients, one is almost inevitably led to the concept of fusion rules, i.e. formal products

$$
A_{i} A_{j}=\sum_{k} N_{i j}^{k} A_{k} .
$$

of primary fields describing the basis-independent content of the operator product algebra.

The matrices $N_{i}$ defined by $\left(N_{i}\right)_{j k}=N_{i j}^{k}$ form themselves a representation of the fusion algebra

$$
N_{i} N_{j}=\sum_{k} N_{i j}^{k} N_{k}
$$


as follows from unitarity of the matrix $S$; this expresses the associativity property of the algebra (2). The relation (1) implies that the matrix $S$ diagonalizes the matrices $N_{i}$ and their eigenvalues are of the form

$$
\gamma_{i}^{(l)}=\frac{S_{i l}}{S_{0 l}}
$$

and obey the sum rules

$$
\gamma_{i}^{(l)} \gamma_{j}^{(l)}=\sum_{k} N_{i j}^{k} \gamma_{k}^{(l)}
$$

The general study of these fusion algebras [3] and their classification have been the object of much work [4], [5], [6].

The numbers

$$
d_{i} \doteq \gamma_{i}^{(0)}=\frac{S_{i 0}}{S_{00}}
$$

appear as statistical dimensions of superselection sectors [7] in algebraic quantum field theory; as square roots of indices for inclusions of von Neumann algebras [14]; as relative sizes of highest weight modules of chiral symmetry algebras in conformal field theory [1]; and in connection with truncated tensor products of quantum groups (see [8] for an accomplished review). According to (5), these numbers obey the statistical dimension sum rules

$$
d_{i} d_{j}=\sum_{k} N_{i j}^{k} d_{k}
$$

which permits us identify $d_{i}$ as a Frobenius eigenvalue of $N_{i}$.

The paper is organized as follows. In the following section we present the fusion matrices and the braid matrices for the chiral $S U(N)_{K}$ models. The conformal dimensions and central charge are calculated from the braid matrices. In the last section we consider the case $K=2$ and the characteristic polynomials of the fusion matrices are calculated.

\section{The chiral $S U(N)_{K}$ models}

\subsection{The fusion matrices}

An important fact is that conformal families can be interpreted as irreducible representations of the chiral algebra. The primary fields of the chiral $S U(N)_{K}$ models can be represented by those Young tableaux for which $\tilde{\lambda}_{1}-\tilde{\lambda}_{N} \leq K, \quad K \geq 2$, where $\tilde{\lambda}_{i}$ is the number of boxes in the $i$ row. Thus, for a given value of $N$, the corresponding Young diagram is a Weyl chamber truncated by the value of $K$.

Let us now associate to each Young tableaux an $(N-1)$-dimensional vector $\Lambda$, defined by $\Lambda=\sum_{i=1}^{N-1} \lambda_{i} e_{i}$, where $\lambda_{i}=\tilde{\lambda}_{i}+N-i$ and $e_{i}, i=1,2, \ldots, N$ are the weights in 
the $N$-dimensional representation of $S U(N)$, satisfying $\sum_{i=1}^{N} e_{i}=0$ and $e_{i} \cdot e_{j}=\delta_{i j}-1 / N$. The components of vector $\Lambda$ satisfy $\lambda_{i} \in \mathcal{N}, \quad \lambda_{1}>\lambda_{2}>\cdots>\lambda_{N-1}>0$ and $N-1 \leq \lambda_{1} \leq N+K-1$.

Proceeding in the $e_{i}$ direction, the next Young tableaux must be obtained from the preceding one by adding a box in the $i$-row. This gives us a composition law (fusion rules) for the vectors (primary fields)

$$
\Lambda \pm e_{i}=\left\{\begin{array}{cc}
\left(\lambda_{1}, \lambda_{2}, \cdots, \lambda_{i} \pm 1, \cdots, \lambda_{N-1}\right) & \text { for } i \neq N \\
\left(\lambda_{1} \mp 1, \lambda_{2} \mp 1, \cdots, \lambda_{N-1} \mp 1\right) & \text { for } i=N
\end{array}\right.
$$

i.e., we have in mind an elementary field $\sigma$ (and its conjugate $\bar{\sigma}$ ) which interpolates according to the fusion rules [9]

$$
[\sigma][\Lambda]=\sum_{i=1}^{N}\left(N_{\sigma}\right)_{\Lambda}^{\Lambda+e_{i}}\left[\Lambda+e_{i}\right], \quad[\bar{\sigma}][\Lambda]=\sum_{i=1}^{N}\left(N_{\bar{\sigma}}\right)_{\Lambda}^{\Lambda-e_{i}}\left[\Lambda-e_{i}\right]
$$

The vector $\phi=(N-1, N-2, \ldots, 2,1)$ (Weyl vector), may be identified with the vacuum sector, which contains a vacuum state. In this case the vector $\sigma=(N, N-2, \ldots, 2,1)$, is identified with the elementary field and $\bar{\sigma}=(N-2, N-3, \ldots, 2,1)$ with its conjugate.

The fusion rules (9) give a natural basis for write the fusion matrix of elementary fields as:

$$
\left(N_{\sigma}\right)_{\Lambda}^{\Gamma}=\sum_{i=1}^{N} \widehat{\delta}_{\Lambda, \Gamma-e_{i}}, \quad\left(N_{\bar{\sigma}}\right)_{\Lambda}^{\Gamma}=\sum_{i=1}^{N} \widehat{\delta}_{\Lambda, \Gamma+e_{i}}
$$

where

$$
\widehat{\delta}_{\Lambda, \Gamma \mp e_{i}} \doteq\left\{\begin{array}{c}
\delta_{\lambda_{1} \gamma_{1}} \delta_{\lambda_{2} \gamma_{2}} \cdots \delta_{\lambda_{i} \gamma_{i} \mp 1} \cdots \delta_{\lambda_{N-1} \gamma_{N-1}}, \text { if } i \neq N \\
\delta_{\lambda_{1} \gamma_{1} \pm 1} \delta_{\lambda_{2} \gamma_{2} \pm 1} \cdots \lambda_{\lambda_{N-1} \gamma_{N-1} \pm 1}, \text { if } i=N
\end{array}\right.
$$

For example, the chiral $S U(2)_{2}$ (Ising) model has three fields, labelled by $\Phi_{\Lambda}: \Phi_{(1)}=$ $\phi$ (vacuum), $\Phi_{(2)}=\sigma$ (spin) and $\Phi_{(3)}=\epsilon$ (energy). From the composition law (8) we can write the fusion rules for the elementary field $\sigma$

$$
[\sigma][\phi]=[\sigma], \quad[\sigma][\sigma]=[\phi]+[\sigma], \quad[\sigma][\epsilon]=[\sigma]
$$

which give the fusion matrix $\left(N_{\sigma}\right)_{\lambda}^{\gamma}=\delta_{\lambda \gamma-1}+\delta_{\lambda \gamma+1}$, and the remaining fusion matrices can be easily derived by the fusion algebra (3):

$$
N_{\phi}=\mathbf{1}, \quad N_{\sigma}=\left(\begin{array}{ccc}
0 & 1 & 0 \\
1 & 0 & 1 \\
0 & 1 & 0
\end{array}\right), \quad N_{\epsilon}=\left(\begin{array}{lll}
0 & 0 & 1 \\
0 & 1 & 0 \\
1 & 0 & 0
\end{array}\right) .
$$

For chiral $S U(3)_{K}$ models we have:

$$
\left(N_{\sigma}\right)_{\Lambda}^{\Gamma} \equiv\left(N_{\sigma}\right)_{\left(\lambda_{1}, \lambda_{2}\right)}^{\left(\gamma_{1}, \gamma_{2}\right)}=\delta_{\lambda_{1} \gamma_{1}-1} \delta_{\lambda_{2} \gamma_{2}}+\delta_{\lambda_{1} \gamma_{1}} \delta_{\lambda_{2} \gamma_{2}-1}+\delta_{\lambda_{1} \gamma_{1}+1} \delta_{\lambda_{2} \gamma_{2}+1}
$$


In the case $K=2$ attend six fields $\Phi_{\Lambda}: \Phi_{(2,1)}=\phi$ (vacuum), $\Phi_{(3,1)}=\sigma, \Phi_{(4,1)}=\bar{\psi}$, $\Phi_{(4,2)}=\epsilon, \Phi_{(4,3)}=\psi$ and $\Phi_{(3,2)}=\bar{\sigma}$. The fusion rules for the elementary field is

$$
\begin{aligned}
& {[\sigma][\phi]=[\sigma], \quad[\sigma][\bar{\psi}]=[\bar{\sigma}], \quad[\sigma][\psi]=[\epsilon]} \\
& {[\sigma][\bar{\sigma}]=[\phi]+[\varepsilon], \quad[\sigma][\sigma]=[\psi]+[\bar{\sigma}], \quad[\sigma][\epsilon]=[\bar{\psi}]+[\sigma] .}
\end{aligned}
$$

and the fusion matrices are

$$
\begin{aligned}
\mathbf{N}_{\sigma}= & \left(\begin{array}{llllll}
0 & 1 & 0 & 0 & 0 & 0 \\
0 & 0 & 1 & 1 & 0 & 0 \\
1 & 0 & 0 & 0 & 1 & 0 \\
0 & 0 & 0 & 0 & 1 & 0 \\
0 & 1 & 0 & 0 & 0 & 1 \\
0 & 0 & 1 & 0 & 0 & 0
\end{array}\right), N_{\psi}=\left(\begin{array}{lllllll}
0 & 0 & 0 & 1 & 0 & 0 \\
0 & 0 & 0 & 0 & 1 & 0 \\
0 & 1 & 0 & 0 & 0 & 0 \\
0 & 0 & 0 & 0 & 0 & 1 \\
0 & 0 & 1 & 0 & 0 & 0 \\
1 & 0 & 0 & 0 & 0 & 0
\end{array}\right), \\
\mathbf{N}_{\epsilon}= & \left(\begin{array}{llllll}
0 & 0 & 0 & 0 & 1 & 0 \\
0 & 1 & 0 & 0 & 0 & 1 \\
0 & 0 & 1 & 1 & 0 & 0 \\
0 & 0 & 1 & 0 & 0 & 0 \\
1 & 0 & 0 & 0 & 1 & 0 \\
0 & 1 & 0 & 0 & 0 & 0
\end{array}\right), N_{\phi}=\mathbf{1}, N_{\bar{\sigma}}=N_{\sigma}^{t}, N_{-}=N_{\psi}^{t},
\end{aligned}
$$

where $N^{t}$ standing for the transposed of $N$.

Inspecting the Young diagrams for a given $N$ and a given value of $K$, one can solve (घ) to get the statistical dimensions associated with each irreducible representation of the $S U(N)_{K}$ models 10, 11]:

$$
d(\Lambda)=\prod_{i=1}^{N-1} \frac{s\left(\lambda_{i}\right)}{s(1)} \prod_{i<j}^{N-1} \frac{s\left(\lambda_{i}-\lambda_{j}\right)}{s(j-i+1)}
$$

where $s(\lambda)=\sin (\pi \lambda /(N+K))$. In particular, $d(\phi)=1$ and $d(\sigma)=s(N) / s(1)$. The statistical dimensions of conjugate representations coincide.

\subsection{The exchange algebra}

Due to the non-additivity of conformal scale dimensions, the spectrum decomposition of local fields $\Phi(\vec{x})$, with respect to the center of the conformal group is non-trivial[13]:

$$
\Phi(\vec{x})=\sum_{\eta} \Phi_{\eta}(\vec{x})
$$

where every $\Phi_{\eta}(\vec{x})$ is a non-local object with $\eta$-dependent complex phases that occur in the special conformal transformation laws. The range of the label $\eta$ is determined by the selection rules of scale dimensions. 
Applied to the vacuum state, the fields of a conformal block $[\alpha]$ generate a representation sector $\mathcal{H}_{\alpha}$ of the stress-energy tensor field. Applied to a state in $\mathcal{H}_{\beta}$, fields of a conformal block $[\alpha]$ give us contributions in all space $\mathcal{H}_{\gamma}$ allowed by the fusion rules. Introducing orthogonal projectors $P_{\beta}$ on the sectors $\mathcal{H}_{\beta}$ one obtains the decomposition

$$
\Phi^{\alpha}(\vec{x})=\sum_{\beta, \gamma} P_{\gamma} \Phi^{\alpha}(\vec{x}) P_{\beta} \equiv\left(\Phi^{\alpha}\right)_{\gamma \beta}(\vec{x}) .
$$

This decomposition coincides with the spectral decomposition (14) with the previous label $\eta$ replaced by " fusion channels " for the "charge " $\alpha$.

It is a well established fact that conformal field theories can be constructed on Hilbert spaces which are direct sums of irreducible representations of an observable algebra $\mathcal{L} \oplus \overline{\mathcal{L}}$. Both subalgebras $\mathcal{L} \oplus \mathbf{1}$ and $\mathbf{1} \oplus \overline{\mathcal{L}}$ is associated to one light-cone. We also add the further requirement that the Hilbert space contains only a finite number of irreducible representations of $\mathcal{L}$ and $\overline{\mathcal{L}}$. Hence

$$
\mathcal{H}=\oplus_{\alpha, \bar{\alpha}} \mathcal{H}_{\alpha} \otimes \mathcal{H}_{\bar{\alpha}}
$$

where $\mathcal{H}_{\alpha}\left(\mathcal{H}_{\bar{\alpha}}\right)$ are irreducible representations of $\mathcal{L}(\overline{\mathcal{L}})$ and the pair $(\alpha, \bar{\alpha})$ takes its values in a finite set.

Due to the light-cone factorization of the stress-energy tensor field algebra, the label $[\alpha]$ of conformal blocks are in fact pairs $\left[\alpha_{+}, \alpha_{-}\right]$. Both representation sectors and the projectors factorize into the projected fields

$$
\left(\Phi^{\alpha}\right)_{\gamma \beta}(\vec{x})=\left(A^{\alpha_{+}}\right)_{\gamma_{+} \beta_{+}}\left(\vec{x}_{+}\right) \otimes\left(A^{\alpha_{-}}\right)_{\gamma_{-} \beta_{-}}\left(\vec{x}_{-}\right) .
$$

Finally the monodromy properties of the conformal blocks are equivalent to the exchange algebra on either light-cone

$$
\left(A^{\alpha_{1}}\right)_{\delta \gamma}(x)\left(A^{\alpha_{2}}\right)_{\gamma \beta}(y)=\sum_{\gamma^{\prime}}\left[R_{\left(\alpha_{1}, \alpha_{2}\right)}^{(\delta, \beta)}(s)\right]_{\gamma \gamma^{\prime}}\left(A^{\alpha_{2}}\right)_{\delta \gamma^{\prime}}(y)\left(A^{\alpha_{1}}\right)_{\gamma^{\prime} \beta}(x) .
$$

Here (and from now on ) we have omitted the indices " \pm ". The numerical structure constants $R$ are matrices which satisfy three basic properties (see [12):

(i) $\left[R_{\left(\alpha_{1}, \alpha_{2}\right)}^{(\delta, \beta)}(s)\right]$ depend on $x$ and $y$ only through their relative position. This follows from translation and scale variance. Moreover, if $s=\operatorname{sign}(x-y)= \pm$, then

$$
\left[R_{\left(\alpha_{1}, \alpha_{2}\right)}^{(\delta, \beta)}(+)\right]^{-1}=\left[R_{\left(\alpha_{1}, \alpha_{2}\right)}^{(\delta, \beta)}(-)\right]
$$

(ii) Phase condition: $R_{\left(\alpha_{1}, \alpha_{2}\right)}^{(\delta, \beta)}$ and $R_{\left(\alpha_{2}, \alpha_{1}\right)}^{(\delta, \beta)}$ are related through the following relation

$$
\sum_{\gamma^{\prime}}\left[R_{\left(\alpha_{1}, \alpha_{2}\right)}^{(\delta, \beta)}(s)\right]_{\gamma \gamma^{\prime}}\left[R_{\left(\alpha_{2}, \alpha_{1}\right)}^{(\delta, \beta)}(s)\right]_{\gamma^{\prime} \gamma^{\prime \prime}} \exp \left(2 i \pi\left(h_{\gamma}+h_{\gamma^{\prime}}-h_{\delta}-h_{\beta}\right)=\delta_{\gamma, \gamma^{\prime \prime}} .\right.
$$

Where $h_{\gamma}$ 's are primary dimensions of the representations $[\gamma]$. This follows from invariance under special conformal transformation. 
(iii) Braid relations: The exchange matrices satisfy

$$
\begin{aligned}
& \sum_{\beta_{1}^{\prime \prime}}\left[R_{\left(\alpha_{1}, \alpha_{2}\right)}^{\left(\beta_{0}, \beta_{2}\right)}(s)\right]_{\beta_{1} \beta_{1}^{\prime \prime}}\left[R_{\left(\alpha_{1}, \alpha_{3}\right)}^{\left(\beta_{1}^{\prime \prime}, \beta_{3}\right)}(s)\right]_{\beta_{2} \beta_{2}^{\prime \prime}}\left[R_{\left(\alpha_{2}, \alpha_{3}\right)}^{\left(\beta_{0}, \beta_{2}^{\prime}\right)}(s)\right]_{\beta_{1}^{\prime \prime} \beta_{1}^{\prime}}= \\
& \sum_{\beta_{2}^{\prime \prime}}\left[R_{\left(\alpha_{2}, \alpha_{3}\right)}^{\left(\beta_{1}, \beta_{2}\right)}(s)\right]_{\beta_{2} \beta_{2}^{\prime \prime}}\left[R_{\left(\alpha_{1}, \alpha_{3}\right)}^{\left(\beta_{0}, \beta_{2}^{\prime \prime}\right)}(s)\right]_{\beta_{1} \beta_{1}^{\prime}}\left[R_{\left(\alpha_{1}, \alpha_{2}\right)}^{\left(\beta_{1}^{\prime}, \beta_{3}\right)}(s)\right]_{\beta_{2}^{\prime \prime} \beta_{2}^{\prime}},
\end{aligned}
$$

which is the consistency relation for the associativity of the exchange algebra (18).

All these relations were derived in [14] from the theory of localized endomorphism without invoking conformal invariance.

The solution of eq.(21) for all admissible (i.e. consistent with the fusion rules (9) ) indices being irreducible representations of $S U(N)_{K}$ and $\alpha_{1}=\alpha_{2}=\alpha_{3}=\sigma$, "elementary field" [16, is given by

$$
\begin{aligned}
& {\left[R_{(\sigma, \sigma)}^{\left(\Lambda+e_{k}+e_{s}, \Lambda\right)}(+)\right]_{\Lambda+e_{k}, \Lambda+e_{s}}=\eta q^{\frac{1}{2}}\left\{\frac{s\left(\lambda_{k}-\lambda_{s}+1\right) s\left(\lambda_{k}-\lambda_{s}-1\right)}{s\left(\lambda_{k}-\lambda_{s}\right)^{2}}\right\}^{1 / 2}} \\
& {\left[R_{(\sigma, \sigma)}^{\left(\Lambda+e_{k}+e_{s}, \Lambda\right)}(+)\right]_{\Lambda+e_{k}, \Lambda+e_{k}}=\eta q^{\frac{1-\lambda_{k}+\lambda_{s}}{2}}\left\{\frac{s(1)}{s\left(\lambda_{k}-\lambda_{s}\right)}\right\}, \text { for } k \neq s} \\
& {\left[R_{(\sigma, \sigma)}^{\left(\Lambda+2 e_{k}, \Lambda\right)}(+)\right]_{\Lambda+e_{k}, \Lambda+e_{k}}=-\eta q}
\end{aligned}
$$

where $\Lambda=\left(\lambda_{1}, \lambda_{2}, \ldots, \lambda_{N-1}\right), s(\lambda)=\sin (\pi \lambda /(N+K)), q=\exp \left(-\frac{2 i \pi}{N+K}\right)$ and $\eta$ is a arbitrary phase factor which will be fixed latter.

\subsection{The dimensional trajectories and central charge}

The $R$ matrices (22) have been constructed as solution of the braid relations (21) for

the elementary field $\sigma$, of the chiral $S U(N)_{K}$ models. They must also solve the phase condition (20). This yields constraints

$$
\eta^{-2 N}=q^{N+1}
$$

as well as the following equations for the dimensional trajectories

$$
\begin{aligned}
& \exp \left\{2 i \pi\left(2 h_{\Lambda+e_{k}}-h_{\Lambda}-h_{\Lambda+2 e_{k}}\right)\right\}=\eta^{-2} q^{-2}, \\
& \exp \left\{2 i \pi\left(2 h_{\Lambda+e_{k}}-h_{\Lambda}-h_{\Lambda+e_{k}+e_{s}}\right)\right\}=\eta^{-2},
\end{aligned}
$$

for $\lambda_{k}-\lambda_{s}=1$. We have two furthermore equations when $\left|\lambda_{k}-\lambda_{s}\right|=2$, which are

$$
\begin{array}{ll}
\exp \left\{2 i \pi\left(2 h_{\Lambda+e_{k}}-2 h_{\Lambda+e_{s}}\right)\right\} & =q^{-2\left(\lambda_{k}-\lambda_{s}\right)}, \\
\exp \left\{2 i \pi\left(2 h_{\Lambda+e_{k}}-h_{\Lambda}-h_{\Lambda+e_{k}+e_{s}}\right)\right\} & =\eta^{-2} q^{-\left(\lambda_{k}-\lambda_{s}\right)-1} .
\end{array}
$$

Eq.(23) together with the normalized 2-point function of the elementary field allows us to choose among all possible solutions, one that fixes the value of the phase factor, $\eta$, to be related with the conformal dimension of the elementary field $\sigma$ :

$$
\eta=\exp \left\{-\frac{2 i \pi}{N-1} h_{\sigma}\right\}
$$


Next, using the Kac-determinante [15], we assume that the conformal spectrum of the chiral $S U(N)_{K}$ models can be derived from (24 and 25) by the ansatz

$$
h_{\Lambda}=\sum_{k=1}^{N-1}\left(a_{k} \lambda_{k}^{2}+b_{k} \lambda_{k}\right)+\sum_{k<s}^{N-1} c_{k s} \lambda_{k} \lambda_{s}+d
$$

where $a_{k}, b_{k}, c_{k s}$ and $d$ are functions of $N$ and $K$.

Therefore the primary dimensions $(\bmod Z)$ of the irreducible representations of the chiral $S U(N)_{K}$ models [16 can be written as:

$$
\begin{aligned}
h_{\Lambda}= & \frac{(N+K-1)(N-1)}{2 N(N+K)} \sum_{i=1}^{N-1} \lambda_{i}^{2}-\frac{(N+K-1)}{N(N+K)} \sum_{i<j}^{N-1} \lambda_{i} \lambda_{j} \\
& +\sum_{i=1}^{N-1}\left(i-\frac{N+1}{2}\right) \lambda_{i}+\frac{(N+K+1) N\left(N^{2}-1\right)}{24(N+K)}
\end{aligned}
$$

It is also easy to verify that the $h_{\Lambda}$-dimensions enjoy the symmetry

$$
h_{\left(\lambda_{1}, \lambda_{2}, \cdots, \lambda_{N-1}\right)}=h_{\left(\lambda_{1}, \lambda_{1}-\lambda_{N-1}, \lambda_{1}-\lambda_{N-2}, \cdots, \lambda_{1}-\lambda_{2}\right)}
$$

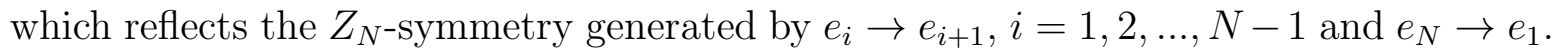
It means that the conformal dimension of conjugate representations coincide.

According to references [14, 7] we introduce the statistics phase $\omega(\Lambda)$, which generalizes the distinction between bosons and fermions of para-statistics, and putting $\alpha=\sum d^{2}(\Lambda) \omega^{-1}(\Lambda)$ one can define the matrices $S$ and $T$ by:

$$
\begin{aligned}
& S_{\Lambda \Gamma}=\frac{1}{|\alpha|} \sum_{\Lambda^{\prime}} N_{\Lambda \Gamma}^{\Lambda^{\prime}} \frac{\omega(\Lambda) \omega(\Gamma)}{\omega\left(\Lambda^{\prime}\right)} d\left(\Lambda^{\prime}\right), \\
& T=\left(\frac{\alpha}{|\alpha|}\right)^{1 / 3} \operatorname{Diag}(\omega(\Lambda)),
\end{aligned}
$$

which satisfy

$$
\begin{aligned}
& S S^{\dagger}=T T^{\dagger}=\mathbf{1}_{N}, \quad T S T S T=S, \\
& S^{2}=C, \quad C T=T C=T,
\end{aligned}
$$

where $C_{\Lambda \Gamma}=\delta_{\bar{\Lambda} \Gamma}$ is the conjugation matrix. This algebra is famous from RCFT but, as observed in [14], it does not depend on any covariance or modular properties.

For the chiral $S U(N)_{K}$ models, the statistics phase is defined by $\omega(\Lambda)=\exp \left(2 i \pi h_{\Lambda}\right)$ (Spin-Statistics Theorem 14]), where $h_{\Lambda}$ is the conformal dimension of the primary field $\Lambda$ given by (28)

Invariance under $S L(2, C)$ transformations allows one to derive from eq.(31) an interesting relation between the central charge and the statistical dimensions [7]:

$$
\exp \left(2 i \pi \frac{c}{8}\right)=\frac{\sum_{\Lambda} d^{2}(\Lambda) \exp \left(2 i \pi h_{\Lambda}\right)}{\sqrt{\sum_{\Lambda} d^{2}(\Lambda)}} .
$$


Substituting (13) and (28) into (31) we get the central charge $(\bmod Z)$ for $S U(N)_{K}$ models

$$
c=(N-1)\left(1-\frac{N(N+1)}{(N+K)(N+K-1)}\right) \text {. }
$$

\section{The $S U(N)_{2}$ models}

At the level $K=2$ this central charge (34) reduces to $c=2(N-1) /(N+2)$ and the primary fields $\Lambda$ are identified with the order fields $\sigma_{k}, k=1,2, \ldots, N-1, Z_{N}$-neutral fields $\epsilon^{(j)}, j=1,2, \ldots \leq N / 2$ and the parafermionic currents $\Psi_{k}, k=0,1, \ldots, N-1$, in Zamolodchikov-Fateev's parafermionic theories [17]. For each $\Lambda$-field we define a "charge $" \quad \nu=\sum_{i=1}^{N-1} \lambda_{i}-N(N-1) / 2 \bmod 2 N$, and collect these $N(N+1) / 2$ primary fields in $N$ cominimal equivalence classes [18], $\left[\phi_{k}^{k}\right], k=0,1, \ldots, N-1$, according to their statistical dimensions (13):

$$
\begin{aligned}
& d_{k}=\prod_{i=0}^{k-1} \frac{s(N-i)}{s(i+1)}, \quad s(x)=\sin \left(\frac{x \pi}{N+2}\right) \\
& d_{0}=1, \quad d_{N-k}=d_{k}, \quad k=1,2, \ldots, N-1
\end{aligned}
$$

$S U(N)_{2}$ representations of the order fields $\phi_{k}^{k}, k=1, \ldots, N-1$ are the fully antisymmetric Young tableaux with $k$ boxes. Tableaux of fields comprising a cominimal equivalence class $\phi_{\nu}^{k}$ in which the representation $\phi_{k}^{k}$ appears, $(\nu=k \bmod 2$, i.e., $\nu=k, k+2, \cdots, 2 N-2-k)$, are obtained by adding $(\nu-k) / 2$ rows of width 2 to the top of the reduced tableau of $\phi_{k}^{k}$.

These equivalence classes are generated by $Z_{N}$ symmetry which connect the representations belonging to each class through of the fusion rules 19

$$
\phi_{\nu_{1}}^{k_{1}} \times \phi_{\nu_{2}}^{k_{2}}=\sum_{k=\left|k_{1}-k_{2}\right| \bmod 2}^{\min \left(k_{1}+k_{2}, 2 N-k_{1}-k_{2}\right)} \phi_{\nu_{1}+\nu_{2}}^{k}
$$

In particular, the elementary field $\phi_{1}^{1},\left(\phi_{1}^{1} \times \phi_{\nu}^{k}=\phi_{\nu+1}^{k-1}+\phi_{\nu+1}^{k+1}\right)$ connects the equivalence class of $\phi_{\nu}^{k}$ with adjacent classes, while the field $\phi_{2}^{0},\left(\phi_{2}^{0} \times \phi_{\nu}^{k}=\phi_{\nu+2}^{k}\right)$, connects the fields in the same cominimal equivalence class. Thus, the chiral $S U(N)_{2}$ fusion algebra can be generated by these two fields. For example, the 6 primary fields of $S U(3)_{2}$ can be collected in 3 cominimal equivalence classes ( modulo the identification $\phi_{\nu}^{k}=\phi_{N+\nu}^{N-k}$ ) as:

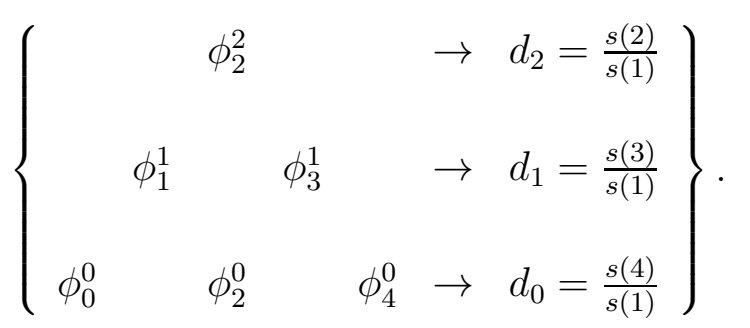




\subsection{The characteristic polynomials}

The fusion rule for the field $\phi_{2}^{0}$ with any primary field $\phi_{\nu}^{k}$ has only one term on the right-hand side. Such fields indicate that the fusion rules can be naturally represented as the chiral ring of some perturbed topological Landau-Ginzburg theory [20], and the correspondent "potential " is obtained by integrate some constraint equations 21], $P_{i}\left(x_{1}, x_{2}, \ldots, x_{n}\right)=0$. If there exists at least one fusion matrix non degenerate, $N_{f}$, i.e., with non degenerate eigenvalues, any fusion matrix may be written as $N_{i}=P_{i}\left(N_{f}\right)$. The matrix $N_{f}$, on the other hand, satisfies its characteristic equation $P(x)=0$, that is also its minimal equation 222. The constraint on $N_{f}$ is thus $P\left(N_{f}\right)=0$ that can be integrated to yield a "potential ".

Here we proceed to the explicit computation of the characteristic polynomials $P_{\nu}^{k}(x)=\operatorname{det}\left(x \mathbf{1}-N_{\phi_{\nu}^{k}}\right)$ associated with the primary fields of the $S U(3)_{2}$ model. Using the fusion matrices $N_{\phi_{\nu}^{k}}$, given by (12) we get:

$$
\begin{aligned}
& P_{0}^{0}(x)=(x-1)^{6} \\
& P_{2}^{0}(x)=P_{4}^{0}(x)=x^{6}-2 x^{3}+1 \\
& P_{1}^{1}(x)=P_{2}^{2}(x)=x^{6}-4 x^{3}-1 \\
& P_{3}^{1}(x)=x^{6}-3 x^{5}+5 x^{3}-3 x-1
\end{aligned}
$$

Now we introduce the numbers

$$
d_{k}(n)=\frac{\sin \left(\frac{n(4-k) \pi}{5}\right)}{\sin \left(\frac{n \pi}{5}\right)}, \quad k=0,1,2, \quad n=1,2 .
$$

to write (38) in a factored form

$$
\begin{aligned}
& P_{2}^{0}(x)=P_{4}^{0}(x)=\left(x^{3}-d_{0}^{3}(1)\right)\left(x^{3}+d_{0}^{3}(2)\right) \\
& P_{1}^{1}(x)=\left(x^{3}-d_{1}^{3}(1)\right)\left(x^{3}-d_{1}^{3}(2)\right) \\
& P_{3}^{1}(x)=\left(x-d_{1}(1)\right)^{3}\left(x-d_{1}(2)\right)^{3} \\
& P_{2}^{2}(x)=\left(x^{3}-d_{2}^{3}(1)\right)\left(x^{3}+d_{2}^{3}(2)\right) .
\end{aligned}
$$

This construction is extended for all $S U(N)_{2}$ models. For each irreducible representation $\phi_{\nu}^{k}$ we associate a factored characteristic polynomial which depend on the parafermionic charge $\nu$ according to $\frac{N}{\nu}=\frac{p}{q}$ where $p$ and $q$ are positive integers mutually coprime:

$$
\begin{aligned}
P_{\nu}^{k}(x) & =\prod_{n=1}^{\frac{N+1}{2}}\left(x^{p}-d_{k}^{p}(n)\right)^{\frac{\nu}{q}}, \quad \text { if } \quad p \cdot q \text {-odd } \\
P_{\nu}^{k}(x) & =\prod_{n=1}^{\frac{N+1}{2}}\left(x^{p}+(-1)^{n} d_{k}^{p}(n)\right)^{\frac{\nu}{q}}, \quad \text { if } \quad p . q \text {-even }
\end{aligned}
$$


for $N$-odd, and

$$
\begin{aligned}
& P_{\nu}^{k}(x)=\left(x^{p}-d_{k}^{p}(l)\right)^{\frac{\nu}{2 q}} \prod_{n=1}^{\frac{N}{2}}\left(x^{p}-d_{k}^{p}(n)\right)^{\frac{\nu}{q}}, \quad \text { if } \quad p . q \text {-odd } \\
& P_{\nu}^{k}(x)=\left(x^{p}+(-1)^{l} d_{k}^{p}(l)\right)^{\frac{\nu}{2 q}} \prod_{n=1}^{\frac{N}{2}}\left(x^{p}+(-1)^{n} d_{k}^{p}(n)\right)^{\frac{\nu}{q}}, \quad \text { if } \quad p . q \text {-even }
\end{aligned}
$$

where $l=(N+2) / 2$, for $N$-even.

Here we have introduced the numbers

$$
\begin{aligned}
d_{k}(n) & =\frac{\sin \left(\frac{n(N+1-k) \pi}{N+2}\right)}{\sin \left(\frac{n \pi}{N+2}\right)}, \quad k=0,1,2, \ldots, N-1, \\
n & =1,2, \ldots, \leq \frac{N+2}{2}
\end{aligned}
$$

which satisfy the following sum rules

$$
d_{i}(n) d_{j}(n)=\sum_{k}\left(N_{i}\right)_{j}^{k} d_{k}(n) .
$$

At level $K>2$, it is also possible write the characteristic polynomials of the fusion matrices of the chiral $S U(N)_{K}$ models. In particular, for the elementary field $\sigma$, the fusion matrix is given by (10) and we can use (4) and (30) to write the correspondent characteristic polynomial as:

$$
P_{\sigma}(x)=\prod_{\Lambda}\left(x-\frac{\exp \left\{2 i \pi\left(h_{\Lambda}+h_{\sigma}\right)\right\}}{d(\Lambda)} \sum_{k=1}^{N} \frac{d\left(\Lambda+e_{k}\right)}{\exp \left\{2 i \pi\left(\Lambda+e_{k}\right)\right\}}\right),
$$

where $h_{\Lambda}$ is the conformal dimension of the field $\Lambda$, given by (28), and $d(\Lambda)$ its statistical dimension, given by (13). For the other fields, the expression for their characteristic polynomials are more complex.

Acknowledgments. I would like to thank Prof. Roland Köberle for useful discussions.

\section{References}

[1] Verlinde E 1988, Nucl. Phys. B300 [FS22] p 360. Dijkgraaf R and Verlinde E 1988, Nucl. Phys. (Proc. Suppl.) 5B p 87.

[2] Belavin A A, Polyakov A M,Zamolodchikov A B 1984, Nucl. Phys. B241 p 333.

[3] Kawai T 1989 Phys. Lett. B217 p 247.

[4] Caselle M, Ponzano G, Ravanini F 1990 , Phys. Lett. B251 p 260; 1992 Int. J. Mod. Phys. B6 p 2075 . 
[5] Eholzer W 1995, Commun. Math. Phys. 172 p 623.

[6] Gepner D and Kapustin A 1995, Phys. Lett. B349 p 71.

[7] Kastler D, Mebkhout M, and Rehren K.-H, in: The Algebraic Theory of Superselection Sectors. Introduction and Recent Results, Kastler D ed, (World Scientific, Singapore, 1990). Rehren K.-H 1990 Springer Lecture Notes in Physics 370 p 139.

[8] Fuchs J, Quantum Dimensions, CERN-TH.6156/91- Communications in Theoretical Physics (published by the Allahabad Mathematical Society).

[9] Gannon T, 1994, "The Classification of $\widehat{S U}(m)_{k}$ Automorphism Invariants ", hep-th/9408119.

[10] Kac V and Wakimoto M 1988, Adv. Math. 70 p 156.

[11] Fuchs J 1995, "Affine Lie Algebras and Quantum Groups", 2nd eddition, Cambridge University Press.

[12] Rehren K.-H and Schroer B 1989, Nucl. Phys. B312 p 715.

[13] Rehren K.-H and Schroer B 1988, Nucl. Phys. B295 [FS21] p 229.

[14] Fredenhagen K, Rehren K.-H, and Schroer B 1989, Commun. Math. Phys. 125 p 201.

[15] Kac, V G and Petserson D H 1984, Adv. Math. 53 p 125.

[16] Pasquier V 1988, Nucl. Phys. B295[FS21] p 491.

Wenzl H 1988, Inv. Math. 92 p 349. Reshetikhin N Yu, LOMI preprint E-4-87.

[17] Zamolodchikov A B and Fateev V A 1985, Sov. Phys JETP 62 p 215.

[18] Naculich S G, Riggs H A and Schnitzer H 1990, Phys. Lett. B246 p 417.

[19] Gepner D and Qiu Z 1987, Nucl. Phys. B285 p 423.

[20] Intriligator K 1991, Mod. Phys. Lett. 38A p 3543.

[21] Gepner D 1991, Commun. Math. Phys. 141 p 381.

[22] Francesco P Di and Zuber J.-B 1993, J. Phys. A:Math. Gen 26 p 1441. 